\section{$\underset{\substack{\text { hommes } \\ \text { \& migrations }}}{ }$}

\section{Hommes \& migrations}

Revue française de référence sur les dynamiques

migratoires

$1304 \mid 2013$

Frontières

\title{
La gouvernance des flux migratoires
}

"indésirables"

Cas d'étude de Calais et Lampedusa

\section{Marie Bassi et Shoshana Fine}

\section{(2) OpenEdition \\ Journals}

Édition électronique

URL : http://journals.openedition.org/hommesmigrations/2647

DOI : 10.4000/hommesmigrations.2647

ISSN : 2262-3353

Éditeur

Musée national de l'histoire de l'immigration

\section{Édition imprimée}

Date de publication : 1 octobre 2013

Pagination : 77-83

ISBN : 978-2-919040-24-7

ISSN : 1142-852X

Référence électronique

Marie Bassi et Shoshana Fine, "La gouvernance des flux migratoires "indésirables" », Hommes \&

migrations [En ligne], 1304 | 2013, mis en ligne le 01 janvier 2017, consulté le 03 mai 2019. URL : http:// journals.openedition.org/hommesmigrations/2647; DOI : 10.4000/hommesmigrations.2647 
Migrants afghans retournant dans la " jungle "

après une distribution de repas dans le centre de Calais,

France, 2009

( ) Mathias Depardon 


\title{
LA GOUVERNANCE DES FLUX MIGRATOIRES "INDÉSIRABLES" CAS D'ÉTUDE DE CALAIS ET LAMPEDUSA
}

\begin{abstract}
par MARIE BASSI, doctorante en science politique au Centre d'études et de recherches internationales (CERI), et SHOSHANA FINE, doctorante en science politique au Centre d'études et de recherches internationales (CERI).
\end{abstract}

\begin{abstract}
La politique migratoire européenne se fonde sur un modèle dominant qui conçoit la frontière comme un filtre et non comme un mur empêchant toute mobilité. L'étude de Lampedusa et de Calais illustre les différentes techniques utilisées pour contrôler la mobilité des "indésirables" dans les zones frontières. Celles-ci reposent sur un mélange de discours sécuritaires (les immigrés sont une menace) et humanitaires (les immigrés sont des victimes) et impliquent une multitude d'acteurs.
\end{abstract}

\section{Au-delà du national, une gouvernance à plusieurs niveaux}

Depuis la fermeture de l'Union européenne à l'immigration de travail au milieu des années 1970, les demandeurs d'asile et les immigrés en situation irrégulière ont été présentés comme des "indésirables". Leur concentration dans les zones frontières de Calais (France) et Lampedusa (Italie) interroge la gouvernance des frontières de l'UE. Ces frontières symboliques sont des points d'entrée et de sortie de l'espace Schengen ${ }^{1}$ et la forte attention politique et médiatique portée à ces lieux de passage a participé à la vision sécuritaire des flux migratoires. Cette sécurisation des migrations a construit un univers de sens partagé par la plupart des acteurs de la gou- vernance des migrations. Afin d'éclairer le contexte migratoire de Lampedusa et de Calais, nous nous concentrerons sur la gouvernance verticale et horizontale des flux migratoires "indésirables". La dimension verticale se réfère à l'interaction entre les différentes échelles de gouvernement : international, européen, national et local. La migration touche aux attributs fondamentaux de la souveraineté territoriale des États-nations, perçus comme l'unité organisationnelle centrale. La plupart des recherches se sont donc longtemps focalisées sur le niveau national comme dimension clé des politiques d'immigration (l'École de Chicago) et d'in- 
tégration ${ }^{2}$. Ce nationalisme méthodologique a été progressivement remis en question dans le champ des études migratoires (et plus largement dans les sciences sociales). Catherine Wihtol de Wenden ${ }^{3}$ soutient que dans un monde

Les zones frontières de Lampedusa et de Calais sont le résultat de politiques, de pratiques et de discours émanant de différents niveaux,

d'acteurs étatiques et non étatiques, formels et informels, qui sont parfois en tension, mais qui sont unis par une perception partagée des flux comme un problème à résoudre. globalisé, il est impossible d'analyser la migration dans un cadre limité à l'acteur national. Pour intégrer la multitude d'acteurs et de niveaux concernés par la gouvernance de la mobilité, certaines études se sont concentrées sur l'échelle locale ${ }^{4}$ ou européenne ${ }^{5}$.

Plus récemment, des chercheurs ont souligné l'entrée de la question migratoire dans l'agenda mondial'. La dimension verticale de la gouvernance des frontières ne doit pas laisser de côté les interactions horizontales entre des acteurs publics et des acteurs non étatiques. En effet, il est impossible de comprendre la gouvernance des frontières sans prendre en compte les acteurs privés (professionnels de la sécurité, contrôleurs aériens...), les acteurs de la société civile (associations, ONG, Églises, avocats...) et les technologies? Certains acteurs se trouvent physiquement sur des territoires frontaliers, tandis que d'autres agissent à distance à partir de pays tiers ${ }^{8}$. Enfin, Elspeth Guild $^{9}$ insiste sur la position du migrant comme acteur de la gouvernance des migrations.

Les zones frontières de Lampedusa et de Calais sont le résultat de politiques, de pratiques et de discours émanant de différents niveaux, d'acteurs étatiques et non étatiques, formels et informels, qui sont parfois en tension, mais qui sont unis par une perception partagée des flux comme un problème à résoudre. L'imbrication des différents niveaux prend tout son sens dans ces deux cas d'étude qui sont au cœur des préoccupations nationales et européennes en matière de lutte contre l'immigration irrégulière. Ces contextes locaux très médiatisés (arrivées par voie maritime sur les côtes de Lampedusa, centre de Sangatte ou "jungle"10 de Calais) influencent la construction sécuritaire des agendas migratoires nationaux, européens et internationaux.

\section{L'Europe à Lampedusa}

L'entrée de l'Italie dans l'espace Schengen en octobre 1997 contraint le gouvernement italien à renforcer le contrôle de ses frontières afin de rassurer les autres États membres de l'espace Schengen, préoccupés par la perméabilité des frontières italiennes. L'arrivée de nombreux Albanais et de Kurdes sur les côtes méridionales entre 1990 et 1997 est présentée comme une "invasion" par les médias et les hommes politiques. L'intensification des expulsions, le renforcement des contrôles aux frontières, la restriction des canaux d'entrée légaux à travers l'instauration de quotas et la création de centres de rétention par la première grande loi migratoire italienne de 1998 visaient ainsi à satisfaire les exigences des États membres ${ }^{11}$. Dans les années 2000, la mise en scène médiatique des embarcations de fortune venant s'échouer sur les côtes siciliennes a également été un levier pour faire de l'Agence européenne pour la gestion de la coopération opérationnelle aux frontières extérieures des États membres de l'Union européenne (Frontex), créée en 2004,

\footnotetext{
2. Rogers Brubaker, Citizenship and Nationhood in France and Germany, Cambridge, Harvard University Press, 1992 ; Yasemin Soysal, Limits of Citizenship, Chicago, University of Chicago Press, 1994. 3. Catherine Wihtol de Wenden, La Question migratoire au XXIe siècle, Paris, Presses de Sciences Po, 2013. 4. Tiziana Caponio, Maren Borkert The Local Dimension of Migration Policymaking, Amsterdam, Amsterdam University Press, 2010. 5. Virginie Guiraudon (dir.), "Les effets de l'européanisation des politiques d'immigration", in Politique européenne, $n^{\circ}$ 31, 2010 ; Gallya Lahav, Immigration and Politics in the New Europe. Reinventing Borders, Cambridge, Cambridge University Press, 2004. 6. Martin Geiger, Antoine Pecoud, The Politics of International Migration Management, Londres, Palgrave Macmillan, 2010. 7. Claire Rodier, Xénophobie business, Paris, La Découverte, 2012. 8. Virginie Guiraudon, Christian Joppke, Controlling a New Migration World, Londres, Routledge, 2001. 9. Elspeth Guild, Security and Migration in the 21st Century, Cambridge, Polity Press, 2009. 10. Vaste zone proche du port de Calais où des centaines de migrants en transit avaient établi leurs campements de fortune. 11. Evelyne Ritaine,"La fabrique politique d'une frontière européenne en Méditerranée. Le 'jeu du mistigri' entre les États et I'Union", Études du CERI, n 186, Paris, 2012
} 
un rouage indispensable de la politique migratoire européenne $\mathrm{e}^{12}$. Ces zones-frontières jouent donc un rôle crucial dans la politisation de l'immigration en Europe et influencent l'agenda, les pratiques migratoires et les représentations de la migration, audelà de leur contexte local.

\section{La déterritorialisation des frontières}

Dans le contexte local de Calais, l'intensification des flux migratoires à partir de la fin des années 1990 et la construction du centre de Sangatte en 1999 ont joué un rôle essentiel dans la sécurisation des agendas migratoires français et britanniques. Plusieurs accords sont signés entre Londres et Paris pour contrôler les flux traversant la Manche. Le Royaume-Uni restant à l'écart de Schengen, la France était tenue de contrôler les flux qui transitaient par sa frontière maritime avec le Royaume-Uni. Pourtant, elle ne procédait pas aux contrôles requis, ne relevant pas les empreintes digitales de dizaines de milliers de demandeurs d'asile et évitant ainsi qu'ils soient signalisés dans Eurodac, une base de données européenne qui aurait permis au Royaume-Uni de les renvoyer en France. En se soustrayant à ces règles, la France évitait que les immigrés restent en transit à Calais ou qu'ils soient renvoyés sur son territoire. En 2002, le gouvernement français ferme le centre de Sangatte, rejetant des milliers de migrants dans des campements de fortune dans la "jungle" aux alentours de Calais. Pour remédier à la mauvaise volonté française, les autorités britanniques signent en 2003 le traité du Touquet ${ }^{13}$ qui leur permet d'effectuer elles-mêmes les contrôles migratoires en France. En 2009, face aux pressions britanniques (et constatant que le Royaume-Uni compliquait la traversée des migrants sur le territoire français), la France détruit la "jungle" et accepte la multiplication des postes de police britanniques dans le Nord-Pas-de-Calais et l'installation d'outils de détection gérés par les techniciens britanniques. Ces pratiques franco-britanniques s'inscrivent dans une tendance européenne visant à externaliser les frontières, c'est-à-dire à étendre les politiques de contrôle à l'ensemble du parcours migratoire ${ }^{14}$. En délocalisant ses contrôles en France, à Calais, la Grande-Bretagne externalise sa politique migratoire ${ }^{15}$.

\section{Coopération et réadmission, les leviers de la politique des frontières}

Les accords de coopération policière (aide financière aux pays d'origine ou de transit pour renforcer les contrôles de leurs frontières, formations communes...) et de réadmission des migrants irréguliers (les pays signataires s'engagent à accueillir les ressortissants des pays tiers ayant transité par leur territoire) sont des instruments de la politique migratoire menée avec les pays tiers, généralement appelée "externalisation". Les accords bilatéraux entre l'Italie et la Libye sont, à ce titre, emblématiCes pratiques francobritanniques s'inscrivent dans une tendance européenne visant à externaliser les frontières, c'est-à-dire à étendre les politiques de contrôle à l'ensemble du parcours migratoire. ques. Les boat-people à Lampedusa sont devenus les symboles du défi de l'immigration irrégulière et ont été utilisés par les gouvernements italiens pour justifier la signature de plusieurs accords avec la Libye, qui devient un 
partenaire essentiel et gagne une réhabilitation diplomatique qu'elle peinait à obtenir depuis plusieurs années. En août 2008, l'Italie signe un "traité d'amitié, partenariat et collaboration" avec la Libye. Jusqu'alors, aucun accord signé avec la Libye n’avait été rendu public. Ce traité perd son nom d'accord de réadmission (qui reste cependant le pilier) et inclut des négociations économiques, culturelles, politiques et militaires. S'ajoute à cela la "récupération des dommages de l'époque coloniale italienne en Libye". Ce traité mène au quasi-blocage de la frontière libyenne par où passent de nombreux migrants subsahariens ${ }^{16}$. Le renvoi des migrants vers un pays non signataire de la Convention de Genève pose de graves problèmes en matière de violation des droits de l'homme (morts dans les camps libyens, refoulements au milieu du désert...).

Ces accords signés entre la plupart des États membres de l'UE et des pays tiers n'arrêtent pas les Cabanes de fortune dans les dunes de Calais, France (c) Mathias Depardon flux mais modifient les routes migratoires. Après l'accord italo-libyen de 2009, les arrivées à Lampedusa ont énormément diminué (31 250 arrivées en 2008 contre 451 en 2010) ${ }^{17}$. Depuis, la frontière gréco-turque est le principal point d'entrée des migrants.

Ces exemples précis révèlent que la frontière est l'objet de négociations permanentes entre différents acteurs institutionnels auxquelles les migrants résistent et qu'elle se déplace au gré de ces négociations et des stratégies des migrants : la frontière est donc en mouvement.

\section{Repli national face aux révolutions arabes}

L'importance des connexions entre les différents niveaux d'action - le fait qu'un défi local soit aussi un défi national et européen - a trouvé une illustration récente. Bien qu'unis par une perception

commune, les différents acteurs (des différents niveaux) peuvent entrer en conflit. L’arrivée à Lampedusa de plus de 51000 migrants, en majorité tunisiens, après la chute de Ben Ali en janvier 2011, a provoqué de fortes tensions entre les autorités locales, nationales et européennes. En premier lieu, les autorités siciliennes ont accusé le gouvernement central et les autres régions de ne pas faire preuve de solidarité et de laisser la Sicile supporter seule le poids de ces arrivées en refusant d'accueillir une partie des migrants. Elles ont demandé une aide financière aux autorités italiennes et européennes pour gérer le phénomène : la question migratoire est ainsi utilisée par les autorités locales comme un moyen de "chantage". Cette crise a ensuite entraîné des tensions entre l'Italie et l'Europe. Le ministre des Affaires étrangères de l'époque, Franco Frattini, évoque une "Europe inerte", qui n'assume pas ses respon- 
sabilités face à la question de l'immigration et le manque de solidarité intra-européenne. Pour que les migrants à peine débarqués sur son territoire puissent circuler dans l'espace Schengen, Rome accorde, en avril 2011, plus de 20000 permis de séjour temporaires aux Tunisiens, provoquant une polémique avec Paris. Face à l'afflux de Tunisiens essayant de pénétrer en France (principale destination des migrants d'origine tunisienne), Paris en refoule de nombreux à la frontière italo-française, à Vintimille, en violation du traité de Schengen. Les gouvernements français et italien proposent alors de suspendre temporairement les accords de Schengen, invoquant une "mesure exceptionnelle" face à un "contexte exceptionnel", proposition rejetée par la Commission européenne. Cet événement conjoncturel révèle un paradoxe : alors que la migration ne peut être gouvernée uniquement par le cadre national, les États résistent à partager le "fardeau". Évidemment, en fonction des relations de pouvoir entre les États membres, certains sont davantage en mesure de résister que d'autres.

\section{Les acteurs non étatiques dans les zones frontières}

Si la dimension verticale de la gouvernance de ces zones frontières est cruciale, une multitude d'acteurs de la société civile (associations, cours de justice, ONG, Églises...) et les migrants euxmêmes participent, à travers leurs pratiques formelles et informelles, et leurs interactions, à leur gouvernance horizontale.

En dépit de leur hétérogénéité, une grande partie des acteurs non étatiques mobilisés à Lampedusa et à Calais ne remettent pas en cause le rôle de la frontière comme filtre des "indésirables". Ils participent ainsi, consciemment ou non, au renforcement de cette interprétation de la frontière à travers une vision "humanitaire" et apolitique des flux. Nombre d'entre eux se limitent à offrir aux migrants des services socio-sanitaires. Ils se conçoivent comme des protagonistes du welfare qui pallient l'incapacité ou l'absence d'interventions étatiques en utilisant le registre de la subsidiarité et du don. "Il y a des carences énormes de la part de l'État. La plus grande offense que peut ressentir l'État est l'ouverture de ces centres par des particuliers qui, face à cette nécessité sociale, ressentent le besoin d'aider leur prochain" (association italienne anonyme).

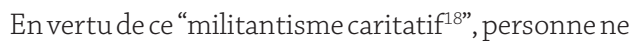
peut être indifférent à la souffrance des "déshérités". Les acteurs utilisent des termes universels qui invitent à la compassion : le "prochain", les "souffrances" et la "misère". Ils prônent un engagement humanitaire en faveur des plus démunis, des "exclus" (chômeurs, malades du sida, SDF, immigrés...). Ils ne procèdent pas à des dénonciations fondées sur des chaînes de responsabilités, relient rarement la situation singu-

lièreà un problèmestructurel et se démarquent d'un militantisme politique.

Ils ne contestent donc pas la vision du migrant "indésirable" comme fardeau ou problème. Bien que ces associations cherchent à se démarquer de la sphère politique, les institutions elles-mêmes les perçoivent souvent Bien que ces associations cherchent à se démarquer de la sphère politique, les institutions elles-mêmes les perçoivent souvent comme des partenaires et utilisent les arguments humanitaires pour justifier leurs politiques (l'insalubrité de la "jungle" justifie son démantèlement). comme des partenaires et utilisent les arguments humanitaires pour justifier leurs politiques (l'insalubrité de la "jungle" justifie son démantèlement). "J'ai beaucoup de contacts avec les associations à Calais : la Belle Étoile, le Secours catholique... Dans un sens, ils sont le bras de l'État français. Ils en reçoivent un financement et ils font un travail qui aurait été fait par l'État au Royaume-Uni (...). Sans ces associations, les migrants chercheraient encore plus à traverser la Manche" (fonctionnaire du ministère des Affaires étrangères britannique).

Le "bras de l'État" se présente de plus en plus souvent sous la forme de grandes organisations 
internationales qui utilisent aussi ce discours humanitaire. Ainsi, l'Organisation internationale pour les migrations (OIM), présente dans les zones frontières, offre un exemple intéressant de cette tendance. L'OIM utilise un langage d'expert et de technocrate et se présente comme un promoteur neutre de pratiques "triple win" selon lesquelles la migration peut bénéficier à tous les acteurs concernés : les États d'accueil, les États d'origine et les migrants ${ }^{19}$. Pourtant, la majorité de ses fonds dépend des États d'accueil, qui financent des projets en cohérence avec leurs agendas sécuritaires. Les campagnes d'information menées par l'OIM auprès des migrants de Calais en font partie. Financées par les Britanniques et l'UE, elles avertissent les migrants des dangers qu'ils encourent en essayant de traverser la Manche et tentent de les persuader d'accepter les programmes de retour volontaire. Elles s'inscrivent dans une tendance sécuritaire qui utilise des arguments humanitaires et préventifs (il faut avertir les migrants des dangers qu'ils encourent en essayant de rejoindre l'Europe). Souvent, les associations ont intériorisé cette logique qu'elles reproduisent dans leurs actions et discours, renforçant ainsi la légitimité de cette approche du contrôle des frontières. Néanmoins, certains acteurs contestent le rôle de la frontière comme filtre et la catégorie des "indésirables” qui en découle. À la différence des précédents, ils cherchent à modifier les pratiques et le discours dominants relatifs à l'immigration.

\section{Résistances collectives : le cas de Lampedusa}

Dans le cas de Lampedusa, le Réseau antiraciste sicilien (RAS) créé en 2004 (jusqu'en 2006) illustre cette résistance. Ce groupe hétérogène comprend des associations culturelles issues de la gauche laïque, des organisations chrétiennes, des avocats et des militants altermondialistes de centres sociaux. Il réclame la fermeture des centres de rétention, dénonce les violations des droits de l'homme qui ont lieu dans ces centres, les expulsions, la criminalisation de l'immigration et la restriction du droit à la mobilité. Derrière ces dénonciations, il conteste l'existence même de la catégorie des migrants "illégaux" et "indésirables" et les pratiques qu'elle entraîne. "Nous considérons les centres de rétention comme illégaux (...). Il n'existe aucune loi en Italie disant que des personnes sans papiers, qui n'ont commis aucun délit (...), doivent être enfermées dans un lieu qui devient une prison" (militant antiraciste à Lampedusa). Bien qu'ils soient minoritaires, ces acteurs remportent quelques victoires qui, sans inverser le paradigme dominant, le délégitiment grâce à des enquêtes journalistiques, des rapports d'ONG ou des décisions juridiques qui soutiennent leurs arguments. À Lampedusa, les actions des militants du RAS ont mené à une condamnation des pratiques italiennes par la Cour européenne des droits de l'homme et par le Parlement européen en 2005.

En effet, en vertu de leur principe fondamental : "Être là où les choses se passent", les militants du RAS se relaient à Lampedusa pour assurer une présence continue sur lîle et témoigner de la situation. Ils réalisent ainsi l'unique témoignage vidéo des expulsions de masse (interdites par le droit international) organisées par le gouvernement Berlusconi entre octobre 2004 et mars 2005. Ces vidéos, envoyées à Amnesty qui les transmet aux institutions européennes, contraignent l'Italie à améliorer ses pratiques et offrent à l'opinion publique une vision critique de la gestion des flux "indésirables".

\section{Résistances individuelles: le cas des migrants à Calais}

En plus de la société civile organisée, les migrants développent des pratiques de résistance. Leur participation à la gouvernance des frontières est illustrée par leurs stratégies face aux règles européennes 
en matière d'asile. Selon la Convention européenne de Dublin, l'État responsable du traitement de la demande d'asile est celui sur lequel le migrant a posé le pied pour la première fois. Pour éviter d'être identifiés et renvoyés dans les pays par lesquels ils sont entrés dans l'UE (Grèce et Italie essentiellement) et qui ont un système d'asile très faible, voire inexistant, les migrants reconstruisent parfois leur identité biographique et biologique. Certains mutilent leurs doigts pour ne pas être identifiés par Eurodac et expulsés ; d'autres empruntent la nationalité de pays où ils ne peuvent être renvoyés (avec lesquels aucun accord de réadmission n'a été signé ou bien là où ils risquent d'être persécutés ${ }^{20}$ ). "Pour survivre à Calais, tu dois jouer avec ton identité. Ici, tout le monde est palestinien; c'est le moyen le plus sûr pour ne pas être expulsé" (Égyptien, trentenaire). "Tu peux faire ça avec de la colle, tu peux brûler tes empreintes avec des clous très chauds ou à l'acide sulfurique... selon moi, ça fait moins mal que d'être renvoyé en Grèce" (Soudanais de 27 ans, Calais).

Bien que ces réactions désespérées puissent être considérées comme des actes de résistance, il s'agit d'actions individuelles qui ne visent pas à faire évoluer les politiques actuelles : les migrants ne contestent pas leur catégorisation d'“indésirables". Pourtant ces actions individuelles contraignent les États à modifier leurs pratiques. Ainsi, la France n'accepte pas d'examiner les demandes d'asile formulées par un migrant aux empreintes illisibles ou lui refuse le statut de réfugié (une pratique informelle, contraire au droit international et national). Les États et les individus sont donc dans une relation d'interaction et de négociation permanente.

\section{Conclusion}

Nous avons montré la pertinence, pour saisir la gouvernance migratoire des zones frontières de Calais et de Lampedusa, d'une approche qui prend en compte l'interaction entre les différents niveaux de gouvernement et les pratiques des acteurs non étatiques. Ces relations s'influencent et prennent des formes différentes : tensions, conflits, délégation, partenariat. La plupart des acteurs partagent une conception de la frontière comme filtre qui restreint la mobilité des "indésirables".

Celle-ci se traduit par des pratiques variées qui se situent sur un continuum allant de l'humanitaire a u sécuritaire. Bien que ce paradigme soit dominant, quelques acteurs non étatiques le contestent ${ }^{21}$. Des militants (pour la plupart issus de la gauche radicale), des organisations chrétiennes, des avocats, des cours de justice, des journalistes et des migrants restreignent la marge de manœuvre des États et de l'UE et participent à la gouvernance des frontières. Ces acteurs défendent un projet politique alternatif basé sur le droit à la mobilité dont les migrants essaient de jouir. Bien que cette position ne soit pas adoptée par les acteurs institutionnels, elle est de plus en plus partagée par les spécialistes des études migratoires ${ }^{22}$ qui définissent la migration comme un fait social ordinaire et inéluctable dans un contexte globalisé. Ils invitent à repenser la frontière afin de déconstruire la catégorie des "indésirables" et pour que tous puissent bénéficier du droit à la mobilité. 